\title{
Z-360 Suppresses Tumor Growth in MIA PaCa-2-bearing Mice via Inhibition of Gastrin-induced Anti-Apoptotic Effects
}

\author{
YOSHIHIRO SHIOMI*, MAKOTO YOSHIMURA*, KAZUMASA KUKI*, YUKO HORI and TAKAO TANAKA \\ Central Research Laboratories, ZERIA Pharmaceutical Co., Ltd., Kumagaya, Japan
}

\begin{abstract}
Background/Aim: The aim of the study was to evaluate the anti-tumor mechanism of Z-360, a gastrin/cholecystokinin-2 receptor (CCK2R) antagonist, in MIA PaCa-2 cells and in a subcutaneous xenograft mice model. Materials and Methods: The anti-tumor effects of Z360 and/or gemcitabine were monitored using a MIA PaCa2 xenograft model. The effect of Z-360 on apoptosis in the model was examined by TUNEL staining and real-time PCR analysis and the effect in MIA PaCa-2 cells stably expressing human CCK2R was also evaluated by caspase-3/7 activity. Results: In this xenograft model, Z-360 significantly reduced the tumor weight, increased TUNEL-positive cells and suppressed the expression of anti-apoptosis factors such as survivin, XIAP and Mcl-1, and these effects of Z-360 combined with gemcitabine were more effective. Furthermore, gastrin-17 and gastrin-34 inhibited apoptosis in vitro and Z-360 dose-dependently abrogated this effect. Conclusion: These results suggest that Z-360 exerts an antitumor effect through a reduction in anti-apoptosis factors by blocking CCK2R.
\end{abstract}

Pancreatic cancer is one of the leading causes of cancerrelated death with a 5-year survival rate of less than $10 \%$ (1, $2)$. It is characterized by a high incidence of loco-regional recurrence and distant metastasis. It is expected that by the year 2030, pancreatic cancer will become the second-most common cause of cancer-related deaths (3). Currently, combination drug therapies with erlotinib or nab-paclitaxel in addition to gemcitabine are commonly used treatments for patients with locally advanced or metastatic disease $(4,5)$.

*These Authors contributed equally to this study.

Correspondence to: Yoshihiro Shiomi, Central Research Laboratories, ZERIA Pharmaceutical Co., Ltd., 2512-1 Numagami, Oshikiri, Kumagaya, Saitama 360-0111, Japan. Tel: +81-48-5363456, Fax: +81 485391072, e-mail: yoshihiro-shiomi@zeria.co.jp

Key Words: Pancreatic cancer, gastrin, cholecystokinin-2 receptor, Z-360, gemcitabine, MiaPaCa-2, xenograft, apoptosis.
Nevertheless, the median survival time is still less than 1 year, which is far from satisfactory. Accordingly, new approaches to chemotherapy are needed. To solve these problems, novel strategies involving less toxic agents that can sensitize pancreatic cancer cells to chemotherapy are necessary.

Tumor development and progression, as well as resistance to most oncologic therapies, result mainly from unresponsiveness of apoptosis pathways (6). The poor prognosis of pancreatic cancer is partly due to intrinsic resistance to the apoptosis-inducing effects of chemotherapy. Therefore, it is important to discover a new drug that attenuates the apoptotic resistance of pancreatic cancer cells.

Apoptosis is triggered by signals from within the cell, such as genotoxic stress, or by extrinsic signals, which involve the activation of cell surface death receptors. The signaling from within the cell, which is also known as the mitochondrial apoptosis pathway, is regulated by suppressor proteins, which include the Bcl-2 family proteins and inhibitor of apoptosis (IAP) proteins. Induced myeloid leukemia cell differentiation protein (Mcl-1) belongs to the Bcl-2 family. Mcl-1 inhibits the activation of caspase-3 following the DNA damage-induced release of cytochrome $c$ from mitochondria. Survivin and X-linked inhibitor of apoptosis protein (XIAP) belong to the IAP family proteins. Survivin and XIAP directly bind to and inhibit caspase-3, -7 and -9 , which are known as apoptosis-inducing factors (7). Previous studies have reported that these anti-apoptotic proteins are overexpressed in pancreatic cancers and might be involved in malignant transformation (8-12).

Cholecystokinin-2 receptor (CCK2R) is a $\mathrm{G}$ proteincoupled receptor for gastrin and cholecystokinin (CCK) which is expressed throughout the body, including the gastrointestinal tract (13). Previous studies have revealed that the malignancy of pancreatic cancer involves CCK2R and gastrin (14-18). Gastrin has several functions, including the secretion of gastric acid and growth of gastrointestinal tissues in the stomach, colon and pancreas through CCK2R $(19,20)$. Gastrin is found in several forms, including gastrin-17, gastrin-34 and progastrin (21). In previous reports, gastrin-17 inhibited apoptosis and stimulated the proliferation of 
pancreatic cancer cells through CCK2R (22-24), and the apoptosis-related factors such as the Bcl-2 and IAP family proteins could be controlled by the gastrin signal pathway (25). CCK2R has therefore been regarded as a therapeutic target for pancreatic cancer (26). CCK2R antagonists such as L-365, 260, JB95008 and Z-360 can inhibit the growth of pancreatic carcinoma-derived cell lines (27-31), suggesting that CCK2R antagonist therapy may be useful for patients with pancreatic cancers (32).

Z-360 (calcium bis [(R)-(-)-3-[3-\{5-cyclohexyl-1-(3,3dimethyl-2-oxo-butyl)-2-oxo-2,3,4,5-tetrahydro- $1 \mathrm{H}$ benzo[b][1,4]diazepin-3-yl\}ureido]benzoate]) is a potent and orally active CCK2R antagonist that was developed by Zeria Pharmaceutical Co., Ltd. In Previous experiments by our group, Z-360 inhibited pentagastrin-stimulated acid secretion in acute fistula rats with $\operatorname{ID}_{50}$ values of $0.17 \mathrm{mg} / \mathrm{kg}$ (i.d.) (33). A radioligand binding assay using $\left[{ }^{3} \mathrm{H}\right] \mathrm{CCK}-8$ demonstrated that $\mathrm{Ki}$ values of $\mathrm{Z}-360$ for CCK1R and CCK2R were $316 \pm 81.2 \mathrm{nM}$ and $0.47 \pm 0.03 \mathrm{nM}$, respectively (30). We previously reported that Z-360 suppressed the proliferation of human pancreatic cancer cell lines in preclinical studies (30), and it was safe and well tolerated when combined with gemcitabine in patients with advanced pancreatic cancer, resulting in clinical benefits in pancreatic carcinoma (34). The impetus for our study was based on current evidence that gastrin plays a crucial role for gastrointestinal tumor growth $(31,34)$. Currently, a phase IIb clinical trial of Z-360 is being conducted in Asia to clarify the interaction between Z-360 and gemcitabine in unresectable advanced pancreatic cancer. Although the antitumor effect of Z-360 has been revealed, the precise mechanism of its anti-tumor action remains to be elucidated.

Here, we investigated the anti-tumor mechanism of Z-360 in the xenograft model of human pancreatic carcinoma MIA PaCa-2. In addition, we examined the effects of Z-360 on gastrin-induced anti-apoptosis in MIA PaCa-2 cells stably expressing human CCK2R. Finally, we discussed the potential of Z-360 as a drug for treating pancreatic cancer.

\section{Materials and Methods}

Reagents. Z-360 was synthesized at the Central Research Laboratories of ZERIA Pharmaceutical Co., Ltd. (Saitama, Japan). Z-360 was dissolved in $0.5 \%$ carboxymethylcellulose sodium solution (CMC-Na) and dimethyl sulfoxide (DMSO) for in vivo tests and in vitro assay, respectively. Gemcitabine hydrochloride (gemcitabine) was purchased from Eli Lilly Japan K.K. (Hyogo, Japan). Human gastrin-17 and gastrin-34 were purchased from Peptide Institute, Inc. (Osaka, Japan) and prepared according to the manufacturer's protocol. Briefly, sterile distilled water or $1 \%$ sodium bicarbonate solution was added to a vial of gastrin-17 or gastrin-34, respectively. Human progastrin was synthesized at Biologica Co., Ltd. (Nagoya, Japan) and sterile distilled water was added to a vial of progastrin.
Cell cultures. The MIA PaCa-2 human pancreatic carcinoma cell line was purchased from the American Type Culture Collection (Manassas, VA, USA). MIA PaCa-2 cells stably expressing human CCK2R (MIA PaCa-2/hCCK2R cells) were obtained from the Central Research Laboratories of ZERIA Pharmaceutical Co., Ltd. MIA PaCa- 2 cells and MIA PaCa-2/hCCK2R cells were cultured using Dulbecco's modified Eagle's medium (DMEM; Thermo Fisher Scientific K.K., Kanagawa, Japan) supplemented with 50 $\mathrm{U} / \mathrm{ml}$ penicillin, $50 \mu \mathrm{g} / \mathrm{ml}$ streptomycin (Thermo Fisher Scientific K.K., Kanagawa, Japan) and 10\% heat-inactivated fetal bovine serum (FBS; Thermo Fisher Scientific K.K., Kanagawa, Japan). Both cell lines were grown as a monolayer in tissue culture flasks at $37^{\circ} \mathrm{C}$ in a humidified atmosphere of $95 \%$ air and $5 \% \mathrm{CO}_{2}$.

Cloning of MIA PaCa-2/hCCK2R cells. Expression vector containing the coding region of human CCK2R was transfected using ViaFect transfection reagent (Promega K.K., Tokyo, Japan) according to manufacturer's protocol. The transfected cells were cultured in culture medium containing $1.25 \mathrm{mg} / \mathrm{ml} \mathrm{G} 418$ (Thermo Fisher Scientific K.K., Kanagawa, Japan), from which a single clone was selected.

Animals. Eight-week-old female BALB/c Slc-nu/nu mice purchased from Japan SLC, Inc. (Shizuoka, Japan) were housed under standard controlled environmental conditions $\left(23 \pm 3^{\circ} \mathrm{C}, 55 \pm 20 \%\right.$ humidity, lights on from 7:00-19:00), with free access to water and standard chow (CRF-1; Oriental Yeast Co., Ltd., Tokyo, Japan). Animals were adapted to the experimental conditions for at least 1 week before the start of experiments. All experimental procedures were approved by the Institutional Animal Care and Use Committee of ZERIA Pharmaceuticals Central Research Laboratories.

Subcutaneous xenograft model. MIA PaCa- 2 cells $\left(3 \times 10^{6}\right.$ cells $)$ were subcutaneously injected into the right flank of the BALB/c nude mice as described previously (30). On Day 14 after the tumor inoculation, the mice were randomly divided into four groups such that animals in each group had an approximately equal average tumor size and body weight. The animals were orally administered Z-360 at a dose of $100 \mathrm{mg} / \mathrm{kg}$ once daily as described previously (30) and intravenously administered gemcitabine at a dose of $50 \mathrm{mg} / \mathrm{kg}$ twice a week for 3 weeks after the inoculation. The tumor size of each animal was measured once a week, and the tumor volume $\left(\mathrm{mm}^{3}\right)$ was calculated by the formula: tumor volume $\left(\mathrm{mm}^{3}\right)=$ length $\times$ width $^{2} \times 1 / 2$. On Day 35 after the tumor inoculation, all the mice were sacrificed, and the tumors were carefully removed and weighed.

Terminal deoxynucleotidyl transferase-mediated dUTP nick end labeling (TUNEL) staining in pancreatic cancer xenografts. TUNEL staining was performed using ApopTag Peroxidase In Situ Apoptosis Detection Kit (EMD Millipore Corporation, MA, USA). Tumor tissues were fixed in formalin and embedded in paraffin. Paraffin-embedded tissues were sectioned $(4 \mu \mathrm{m})$ and deparaffinized. The tissues were treated with $0.001 \%$ proteinase $\mathrm{K}(20 \mu \mathrm{g} / \mathrm{ml})$ for $15 \mathrm{~min}$, then washed with PBS (3 times for $5 \mathrm{~min}$ ) and incubated with PBS containing 3\% hydrogen peroxide for 5 min to block endogenous peroxidase. After 3 washes with PBS, sections were incubated with ApopTag Equilibration Buffer (EMD Millipore Corporation, MA, USA) for $15 \mathrm{~min}$ at room temperature. Sections were incubated with terminal deoxynucleotidyl transferase for $1 \mathrm{~h}$ at $37^{\circ} \mathrm{C}$, and then incubated with ApopTag 
Stop/Wash Buffer (EMD Millipore Corporation, MA, USA) for 20 $\mathrm{min}$ at room temperature. Sections were washed 3 times with PBS and incubated for $30 \mathrm{~min}$ in a solution containing anti-digoxigeninperoxidase. The reaction color was developed by treating tissue sections with 3,3'-diaminobenzidine. The tissues were washed with water and counter-stained with hematoxylin. These processed sections were examined with a fluorescence microscope (BZ-9000; Keyence Corporation, Osaka, Japan). TUNEL-positive cells were quantified by selecting five representative sections per animal and manually counting at 400x magnification. Cell counts from 5 sections were averaged to obtain a single value for each animal. These average values were then used to calculate the mean number of TUNELpositive cells in each experimental group.

RNA isolation. Total RNA was prepared from tumor tissue using Isogen II (Nippon Gene Co., Ltd., Tokyo, Japan). After purification, the amount of RNA was measured by spectrophotometry (OD260). Total RNA $(1 \mu \mathrm{g})$ was converted into first-strand cDNA using the High-Capacity RNA-to-cDNA Kit (Thermo Fisher Scientific K.K. Kanagawa, Japan) according to the manufacturer's protocol.

Quantitative real-time PCR. The first-strand cDNAs were subjected to quantitative real-time PCR with ABsolute QPCR ROX Mix (Thermo Fisher Scientific K.K., Kanagawa, Japan) and TaqMan probes for survivin, X-linked inhibitor of apoptosis (XIAP), induced myeloid leukemia cell differentiation protein (Mcl-1), baculoviral IAP repeatcontaining 2 (BIRC2, also called c-IAP1) and BIRC3 using a real-time thermal cycler (ABI PRISM 7900HT Sequence Detection System; Thermo Fisher Scientific K.K., Kanagawa, Japan). The IDs for TaqMan probes used in this study are survivin, Hs00153353_m1; XIAP, Hs01597783_m1; Mcl-1, Hs00172036_m1; BIRC2, Hs01112284_m1; BIRC3, Hs00154109_m1; and HPRT1, Hs99999909_m1. The program was as follows: $15 \mathrm{~min}$ at $95^{\circ} \mathrm{C}$, and then 45 cycles of $15 \mathrm{~s}$ at $95^{\circ} \mathrm{C}$ and $1 \mathrm{~min}$ at $60^{\circ} \mathrm{C}$. The obtained data were normalized to the expression of hypoxanthine phosphoribosyltransferase1 (HPRT1) and analyzed by the comparative threshold cycle method (Thermo Fisher Scientific K.K., Kanagawa, Japan).

Calcium flux assay. MIA PaCa-2/hCCK2R cells $\left(1.5 \times 10^{4}\right.$ cells/well $)$ were cultured in DMEM containing 10\% FBS for two days after being seeded into 96 well plates. Intracellular $\mathrm{Ca}^{2+}$ release was measured using a Screen Quest ${ }^{\mathrm{TM}}$ Fluo-8 NW Calcium Assay Kit (AAT Bioquest, Inc., Sunnyvale, CA, USA) and a fluid handling integrated fluorescence plate reader (Flex Station; Molecular Devices, LLC., Sunnyvale, CA, USA). Briefly, aliquots of gastrin17 , gastrin-34, progastrin and Z-360 were delivered to the sample plate automatically at the preprogrammed times, and then the fluorescence of Fluo- $8 \mathrm{NW}$ in the culture supernatant was read with excitation at $490 \mathrm{~nm}$ and emission at $525 \mathrm{~nm}$. The $50 \%$ effective dose (ED50) and the 50\% inhibition concentration $\left(\mathrm{IC}_{50}\right)$ values were calculated using GraphPad Prism (GraphPad Software, Inc., La Jolla, CA, USA).

Subculture and pre-treatment of gastrin-17, gastrin-34 and Z-360. MIA PaCa- $/$ hCCK2R cells $\left(1 \times 10^{3}\right.$ cells/well $)$ were seeded in two different 96-well assay plates (Biocoat collagen I, clear or white/clear; Corning International K.K., Tokyo, Japan) with $100 \mu \mathrm{l}$ of culture medium containing $1 \%$ FBS. These assay plates were then incubated in a $\mathrm{CO}_{2}$ incubator $\left(37^{\circ} \mathrm{C}, 5 \% \mathrm{CO}_{2}\right.$, humidified environment).
The cells were allowed to adhere to the assay plates for three days and then the medium was replaced with $50 \mu$ of serum-free medium. After $24 \mathrm{~h}$ serum starvation, $25 \mu \mathrm{l}$ of serum-free medium or Z-360 solution $(1,10$ or $100 \mathrm{nM})$ containing $0.2 \%$ BSA was added to the plates. After $1 \mathrm{~h}$ treatment with Z-360 solution, $25 \mu \mathrm{l}$ of vehicle, gastrin- 17 or gastrin-34 solution $(0.001,0.01,0.1$ or $1 \mathrm{nM}$ ) containing $0.2 \%$ BSA was added to the plates for $24 \mathrm{~h}$ under serum-free conditions.

3-(4,5-dimethylthiazol-2-yl)-5-(3-carboxymethoxyphenyl)-2-(4sulfophenyl)-2H-tetrazolium (MTS) assay. To measure cell viability, MTS assay was performed using MIA PaCa-2/hCCK2R cells. $20 \mu \mathrm{l}$ of Cell Titer 96 Aqueous One Solution (Promega K.K. Tokyo, Japan) was added to each well and incubated at $37^{\circ} \mathrm{C}$ for an additional $120 \mathrm{~min}$ after $24 \mathrm{~h}$ treatment with gastrin solution. The clear plates were then measured at $490 \mathrm{~nm}$ on a microplate reader (SUNRISE Remote; Tecan Japan Co., Ltd., Kanagawa, Japan).

Caspase-Glo 3/7 assay. To monitor cell apoptosis through caspase activation, Caspase-Glo 3/7 assay was performed using MIA PaCa2/hCCK2R cells. $100 \mu \mathrm{l}$ of Caspase-Glo 3/7 Assay Systems (Promega K.K. Tokyo, Japan) was added to each well and incubated at room temperature for more than $20 \mathrm{~min}$ after $24 \mathrm{~h}$ treatment with gastrin solution. The relative light units (RLU) of the white/clear plates were measured on a multilabel counter (ARVO SX; PerkinElmer Japan Co., Ltd., Kanagawa, Japan). The value of caspase-3/7 activity was normalized by the total cell number measured by MTS assay in each group.

Statistical analysis. Results are expressed as the mean \pm standard error of means (SEM). Data were analyzed using statistical analysis software (SAS System Version 9.2; SAS Institute Inc., Cary, NC, USA, and EXSUS Version 8.0.0 and 8.1.0; CAC Croit Corporation, Tokyo, Japan). Time-course data for tumor volume (Figure 1A) were analyzed by a two-way repeated measure analysis of variance (ANOVA). Differences between two groups were assessed using Student's $t$-test. Data involving more than two groups were assessed by Dunnett's test. $p<0.05$ was considered statistically significant.

\section{Results}

Effect of Z-360 on tumor growth in xenograft tumors. We evaluated the effects of Z-360, gemcitabine and the combination of both agents on tumor growth in the MIA $\mathrm{PaCa}-2$ subcutaneous xenograft mice. The animals were orally administered Z-360 at a dose of $100 \mathrm{mg} / \mathrm{kg}$ once daily and intravenously administered gemcitabine at a dose of $50 \mathrm{mg} / \mathrm{kg}$ twice a week for 3 weeks after inoculation. Administration of Z-360 alone or gemcitabine alone slightly reduced tumor volume. In particular, tumor volume in the combination group was significantly lowest in all groups (Figure 1A). On Day 35 after tumor inoculation, the tumor weights of the vehicle, Z-360, gemcitabine and combination groups were $0.24 \pm 0.02 \mathrm{~g}, 0.17 \pm 0.02 \mathrm{~g}, 0.15 \pm 0.01 \mathrm{~g}$ and $0.12 \pm 0.01 \mathrm{~g}$, respectively. Also, tumor weight in the Z-360 alone or gemcitabine alone groups was significantly smaller than that in the vehicle group, and the combination of both agents was most effective (Figure 1B). No significant body 

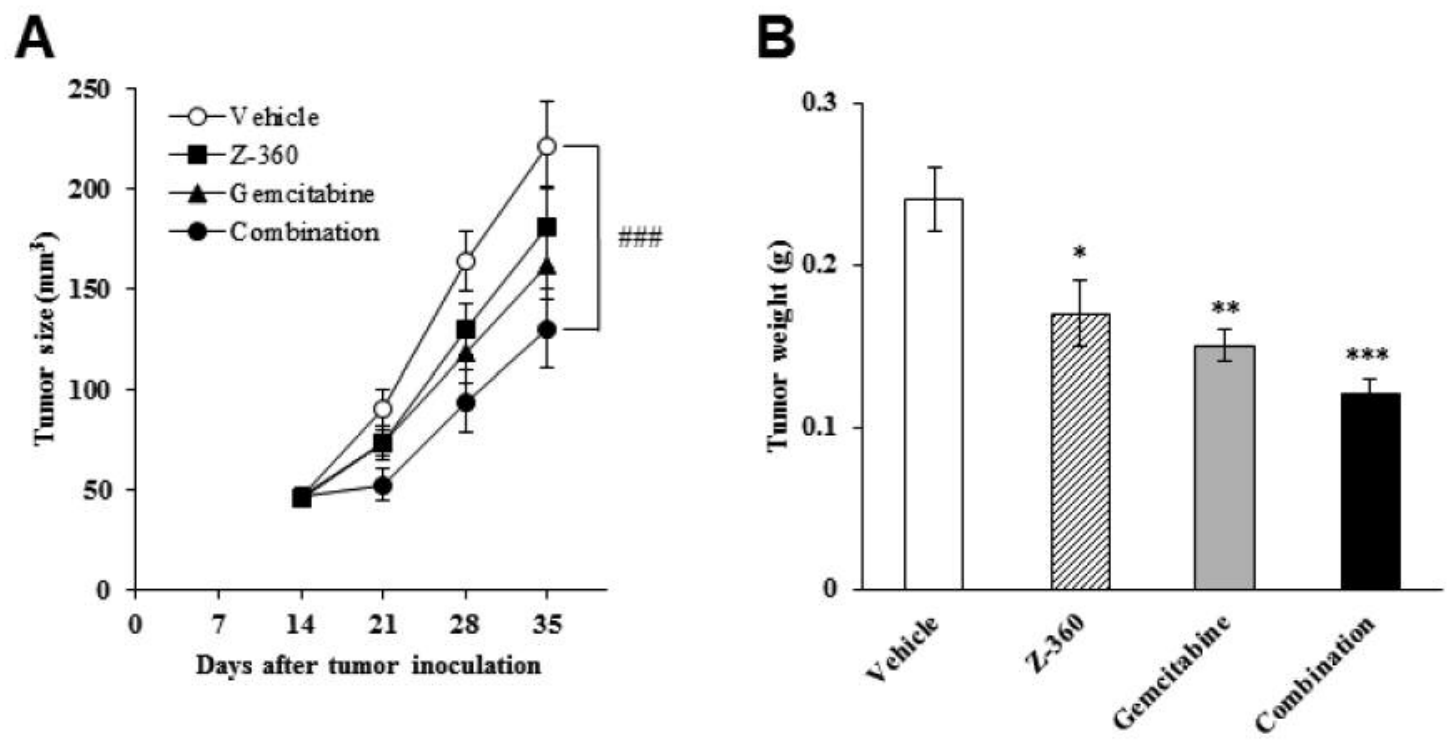

Figure 1. Effect of Z-360 on tumor growth in the MIA PaCa-2 xenograft model. MIA PaCa-2 cells $\left(3 \times 10^{6}\right.$ cells) were injected subcutaneously into

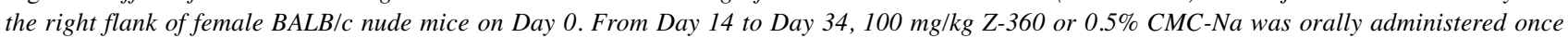
a day. In the same period, $50 \mathrm{mg} / \mathrm{kg}$ gemcitabine or saline was intravenously administered twice a week. (A) Tumor size was measured once a week during this period. Values represent the mean \pm SEM $(n=20)$. On Day 35, the results compared with the vehicle-treated mice were analyzed by twoway repeated measure ANOVA. ${ }^{\# \#} p<0.001$. (B) Tumor weight was measured on Day 35. Values represent the mean $\pm S E M(n=20)$. The results compared with vehicle-treated groups were analyzed by Dunnett's test. ${ }^{*} p<0.05,{ }^{*} p<0.01$ and ${ }^{* * *} p<0.001$.

weight changes were observed in any group (data not shown). These results suggested that Z-360 exerts anti-tumor effect and the combination with gemcitabine suppresses tumor growth more effectively.

Effect of Z-360 on apoptosis in xenograft tumors. On Day 35 , subcutaneous tumor tissues were dissected and the TUNEL assay was performed to assess the effect of Z-360 and/or gemcitabine on apoptosis. Z-360 alone significantly increased TUNEL-positive cells to the same degree as gemcitabine alone and the combination of both agents was more effective (Figure 2). These results suggested that Z-360 shows an anti-tumor effect by inducing apoptosis in tumors and the combination with gemcitabine is more significant.

Effect of Z-360 on anti-apoptotic gene expression in xenograft tumors. To investigate the mechanism of apoptosis in the xenograft tumors, we examined the effects of Z-360 and/or gemcitabine on the expression of anti-apoptosis-related genes. We investigated survivin, XIAP and Mcl-1 as mitochondrial pathway-related genes, and BIRC2 and BIRC3 as death receptor pathway-related genes. The expression of survivin, XIAP and Mcl-1 were significantly down-regulated by the administration of Z-360 alone or gemcitabine alone. The combination of both agents showed additive effects (Figure $3 \mathrm{~A}-\mathrm{C})$. On the other hand, there were no significant changes in BIRC2 or BIRC3 gene expression between the vehicle and Z-360 alone groups (Figure 3D and E). These results suggested that Z-360 prevents tumor growth by regulating the gene expressions of survivin, XIAP and Mcl-1; that is, mitochondrial pathway-related genes of apoptosis and the combination with gemcitabine is more significant.

Agonistic activity of gastrin-17, gastrin-34 and progastrin in MIA PaCa-2/hCCK2R cells. CCK2R releases $\mathrm{Ca}^{2+}$ from endoplasmic reticulum stores according to its activity. To determine the agonistic effect of gastrin-17, gastrin-34 and progastrin against $C C K 2 \mathrm{R}$, we measured intracellular $\mathrm{Ca}^{2+}$ release in MIA $\mathrm{PaCa}-2 / \mathrm{hCCK} 2 \mathrm{R}$ cells. Treatment with gastrin-17 and gastrin-34 up-regulated intracellular $\mathrm{Ca}^{2+}$ release, whereas progastrin $(0.01-10 \mathrm{nM})$ showed no effect on intracellular $\mathrm{Ca}^{2+}$ release (Figure $4 \mathrm{~A}$ ). The $\mathrm{EC}_{50}$ values (95\% confidence intervals) of gastrin-17 and gastrin-34 were $1.5(0.49-4.9)$ and $0.36(0.22-0.60) \mathrm{nM}$, respectively. These results are in agreement with previous reports showing that progastrin did not bind CCK2R, whereas gastrin-17 and gastrin-34 bound CCK2R with high affinity $(35,36)$.

A previous study reported that Z-360 potently inhibited specific binding of $\left[{ }^{3} \mathrm{H}\right] \mathrm{CCK}-8$ to the human CCK2R with a $\mathrm{Ki}$ value of $0.47 \mathrm{nM}$ (30). However, the antagonistic activities of Z-360 for other CCK-2 ligands remain unknown. To determine the antagonistic effect of Z-360 on 
the CCK2R, intracellular $\mathrm{Ca}^{2+}$ release was measured. While $3 \mathrm{nM}$ gastrin-17 and $1 \mathrm{nM}$ gastrin-34 increased intracellular $\mathrm{Ca}^{2+}$ release in MIA PaCa-2/hCCK2R cells, treatment with Z-360 (0.1-10,000 $\mathrm{nM})$ inhibited ligand-induced intracellular $\mathrm{Ca}^{2+}$ release in a concentration-dependent manner (Figure 4B). The $\mathrm{IC}_{50}$ values $(95 \%$ confidence intervals) of Z-360 for $3 \mathrm{nM}$ gastrin-17 and $1 \mathrm{nM}$ gastrin34 were 53 (28-100) and 30 (15-58) nM, respectively. These results demonstrated that Z-360 shows antagonistic activity for the human CCK2R.

Effects of CCK2R ligands on cell number and apoptosis in MIA PaCa-2/hCCK2R cells. MTS assay and Caspase-Glo $3 / 7$ assay were performed to evaluate the anti-apoptosis effects induced by CCK2R ligands in MIA PaCa-2/hCCK2R cells. For the MTS assay, both $1 \mathrm{nM}$ gastrin-17 and $1 \mathrm{nM}$ gastrin-34 significantly increased cell number compared to the vehicle group (Figure 5A and B). In the Caspase-Glo 3/7 assay, both $1 \mathrm{nM}$ gastrin-17 and $1 \mathrm{nM}$ gastrin-34 dramatically reduced caspase-3/7 activity (Figure 5C and D). These findings suggested that both gastrin-17 and gastrin-34 act as CCK2R ligands and exert an anti-apoptotic effect.

Effects of Z-360 on CCK2R ligand-induced apoptosis in MIA PaCa-2/hCCK2R cells. Z-360 dose-dependently inhibited the increase in the total cell number and the decrease in caspase$3 / 7$ activity induced by $1 \mathrm{nM}$ gastrin-17 or $1 \mathrm{nM}$ gastrin-34 (Figure 6). Z-360 at a dose of $100 \mathrm{nM}$ showed significant inhibitory effects against gastrin-17 and gastrin-34. In contrast, Z-360 alone did not show a dose-dependent inhibitory effect on the cell number of MIA PaCa-2/hCCK2R cells (data not shown). The $\mathrm{IC}_{50}$ value of $\mathrm{Z}-360$ was estimated to be approximately $10 \mathrm{nM}$, which was very similar to that of Z-360 for cell proliferation in $\mathrm{CHO}$ cells overexpressing human CCK2R, as previously reported (30).

\section{Discussion}

In the present study, we examined the effects of Z-360 and/or gemcitabine on tumor growth and apoptosis to elucidate the mechanisms of action of Z-360 using the MIA PaCa-2 subcutaneous xenograft mice model. As shown in Figures 1 and 2, Z-360 significantly suppressed tumor weight as previously reported (30), and increased the number of TUNEL-positive cells in the tumor. Furthermore, Z-360 inhibited the gastrin-induced anti-apoptotic effect in vitro (Figures 5 and 6). These results suggest that the anti-tumor effect of Z-360 is related to apoptosis.

Novel therapeutic strategies that have potential to sensitize pancreatic cancer to chemotherapy and increase the efficacy of current treatments for pancreatic cancer are needed. One solution to the problem of drug resistance is to control the
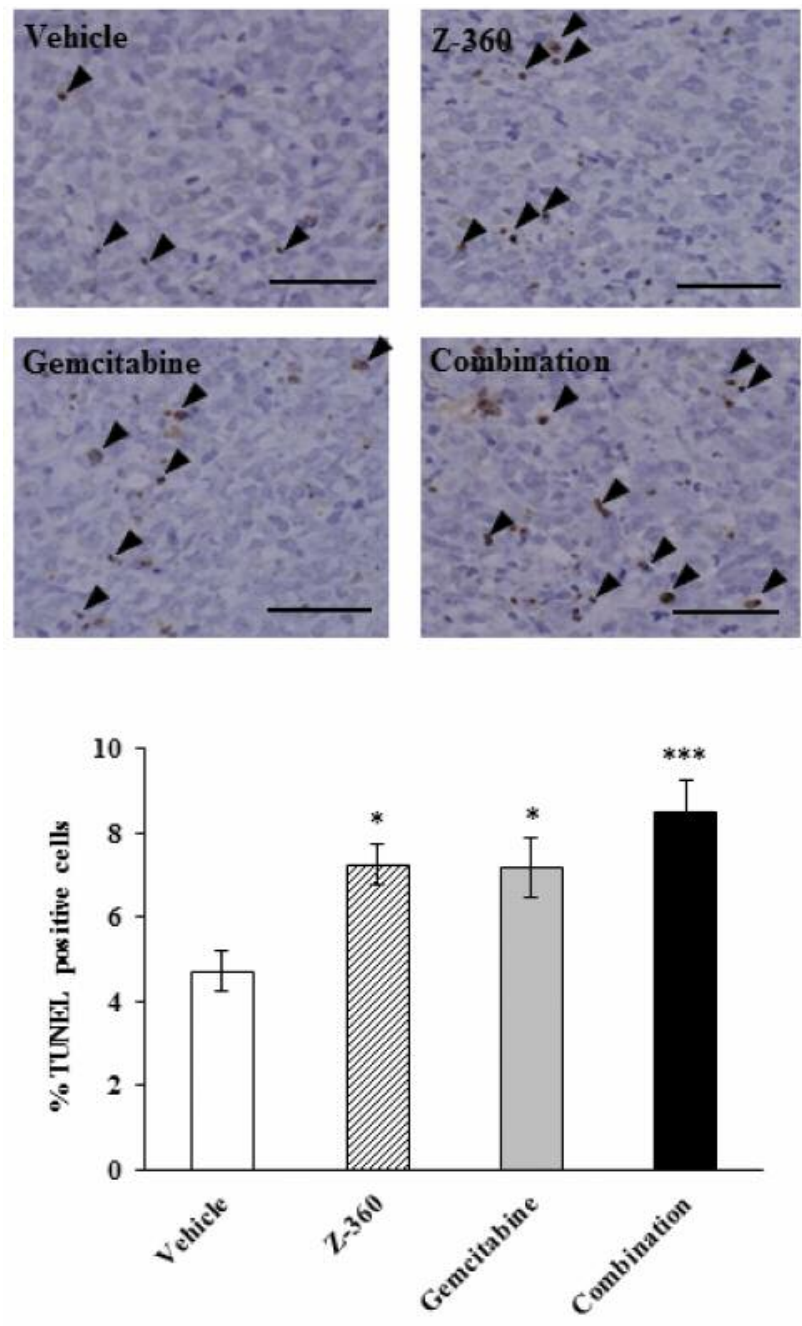

Figure 2. Effects of Z-360 on apoptosis in the MIA PaCa-2 xenograft model. MIA PaCa-2 cells $\left(3 \times 10^{6}\right.$ cells $)$ were injected subcutaneously into the right flank of female BALB/c nude mice on Day 0. From Day 14 to Day 34, $100 \mathrm{mg} / \mathrm{kg} \mathrm{Z}-360$ or $0.5 \% \mathrm{CMC}-\mathrm{Na}$ was orally administered once a day. In the same period, $50 \mathrm{mg} / \mathrm{kg}$ gemcitabine or saline was intravenously administered twice a week. On Day 35, the subcutaneous tumor tissues were dissected and TUNEL staining was performed. Values represent the mean \pm SEM $(n=15)$. The results compared with vehicle-treated groups were analyzed by Dunnett's test. $* p<0.05$, ***p $<0.001$.

apoptosis pathways and then abrogate the resistance to apoptosis, which is a well-known mechanism in a wide range of cancer cells. Resistance to apoptosis is caused by the overexpression of anti-apoptotic factors such as Bcl-2 family proteins and IAP proteins (8-12). We demonstrated that Z360 decreased gene expressions of survivin and XIAP, and Mcl-1 (Figure 3). These results suggested that Z-360 inhibited the expression of apoptosis resistance-related factors. Our previous study showed that Z-360 potently 


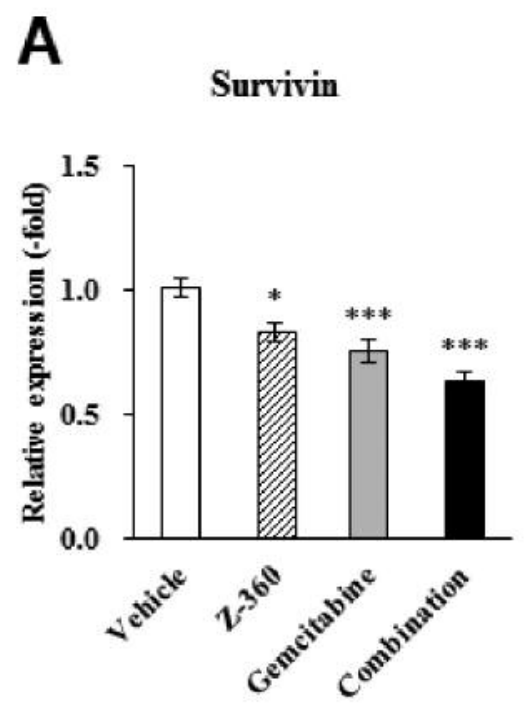

B XIAP

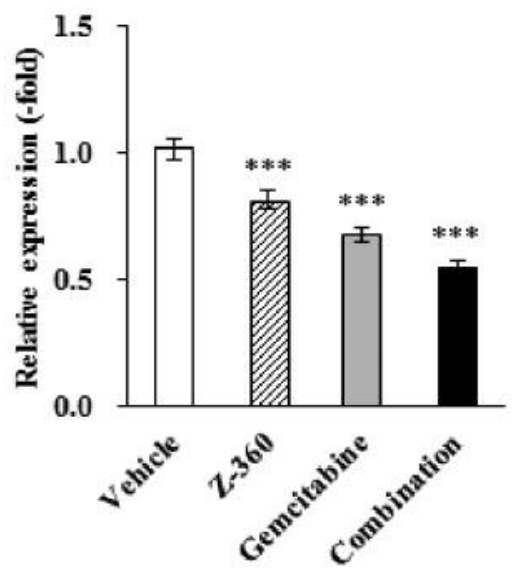

$\mathbf{E}$
BIRC2

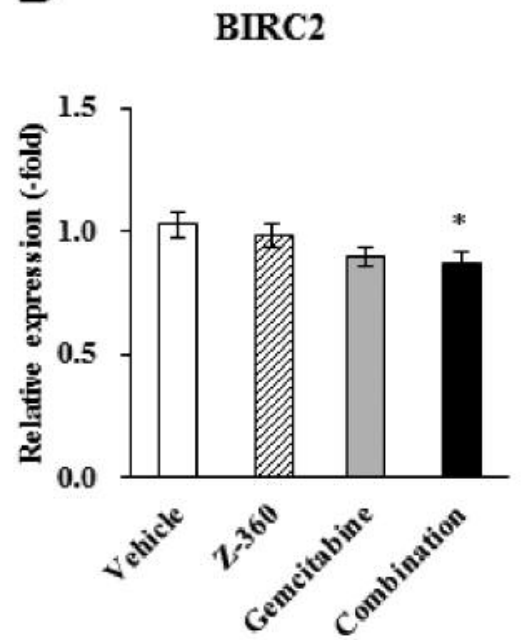

D

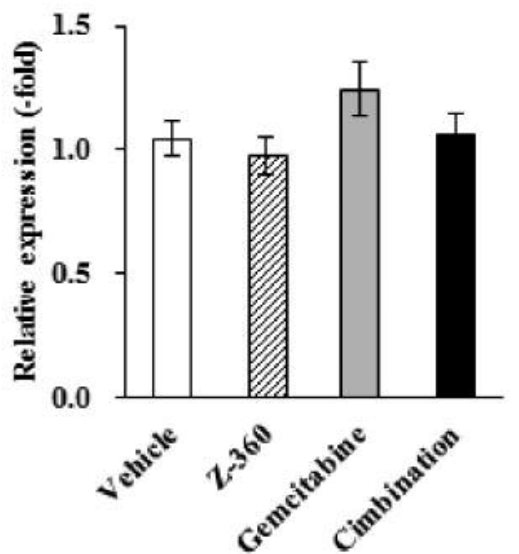

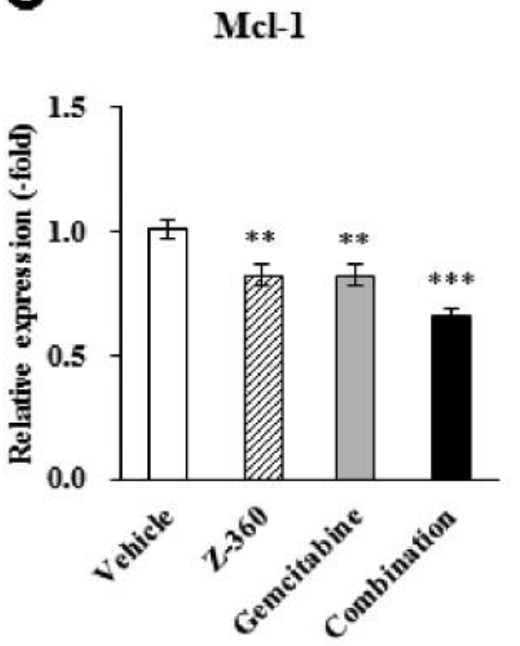

C

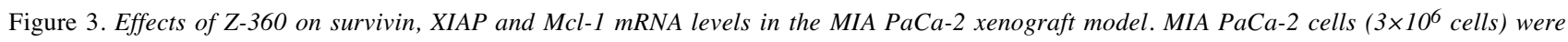
injected subcutaneously into the right flank of female BALB/c nude mice on Day O. From Day 14 to Day 34, Z-360 (100 mg/kg) or 0.5\% CMC-Na was orally administered once a day, and gemcitabine $(50 \mathrm{mg} / \mathrm{kg})$ or saline was intravenously administered twice a week. On Day 35 after implantation, all mice were killed and the tumors were carefully removed for gene expression analyses. Gene expression analyses for (A) survivin, (B) XIAP, (C) Mcl-1, (D) BIRC2 and (E) BIRC3 were performed with quantitative real-time PCR. Data represent fold changes versus the mean for the vehicle group. Values represent the mean \pm SEM $(n=15-18)$. The results compared with vehicle-treated groups were analyzed by Dunnett's test. $* p<0.05, * * p<0.01$ and $* * * p<0.001$.

antagonized the specific binding of $\left[{ }^{3} \mathrm{H}\right] \mathrm{CCK}-8$ to human CCK2R with a $\mathrm{K}_{\mathrm{i}}$ value of $0.47 \mathrm{nM}$, and that Z-360 had a higher affinity for CCK2R than CCK1R, given that the ratio of CCK1R/CCK2R was 615 (30). Previous studies reported that gastrin, as a ligand of CCK2R, increased Mcl-1 and survivin expression $(23,37,38)$. Furthermore, knockdown of CCK2R decreases XIAP protein expression in several human pancreatic cancer cell lines (39). We, therefore, considered that the suppression of survivin, XIAP and Mcl-1 gene expression by Z-360 was through the inhibition of CCK2R.

Previous reports have identified two cell types, each using one of two different CD95 (APO-1/Fas) signaling pathways almost exclusively $(40,41)$. In type I, cells that die through CD95 use the mitochondria-independent apoptosis pathway. In type II, the mitochondrial pathway of apoptosis is employed and anti-apoptosis proteins such as Bcl-2 family proteins protect cells from apoptosis. This study, suggests that treatment of Z-360 alone decreased the expression of 

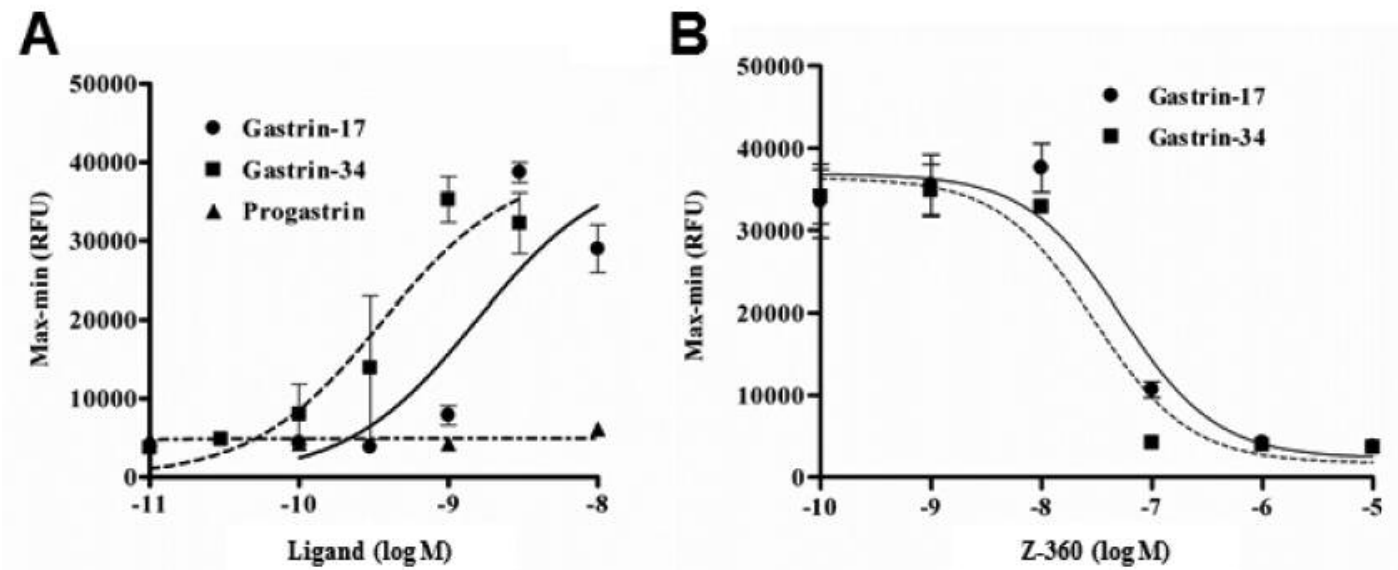

Figure 4. Inhibitory effects of Z-360 on CCK2R ligand-induced intracellular Ca ${ }^{2+}$ release in MIA PaCa-2/hCCK2R cells. (A) The ability of gastrin-17 (solid line), gastrin-34 (dotted line) and progastrin (chain line) to increase the release of intracellular Ca ${ }^{2+}$ was determined using Fluo-8 NW-loaded MIA PaCa-2/hCCK2R cells. Each value represents the mean \pm SEM of data from three independent experiments. (B) The ability of $3 \mathrm{nM}$ gastrin-17 (solid line) and $1 \mathrm{nM}$ gastrin-34 (dotted line) to increase the release of intracellular Ca ${ }^{2+}$ was determined in the presence of various concentrations of Z-360 using Fluo-8 NW-loaded MIA PaCa-2/hCCK2R cells. Each value represents the mean \pm SEM of data from three independent experiments.

A

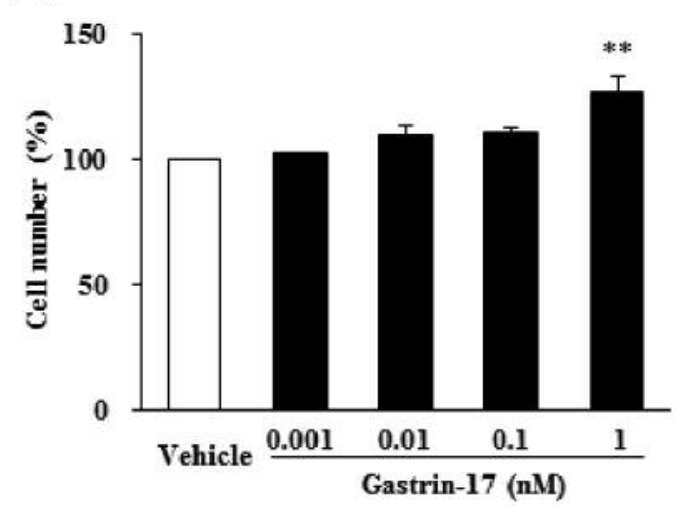

C

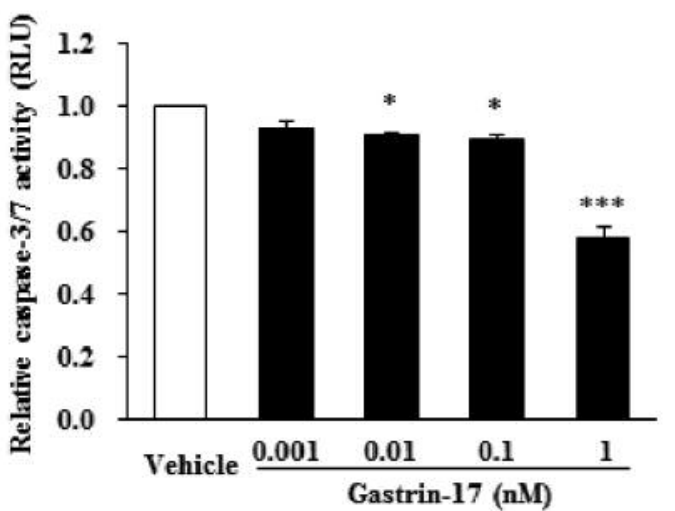

B

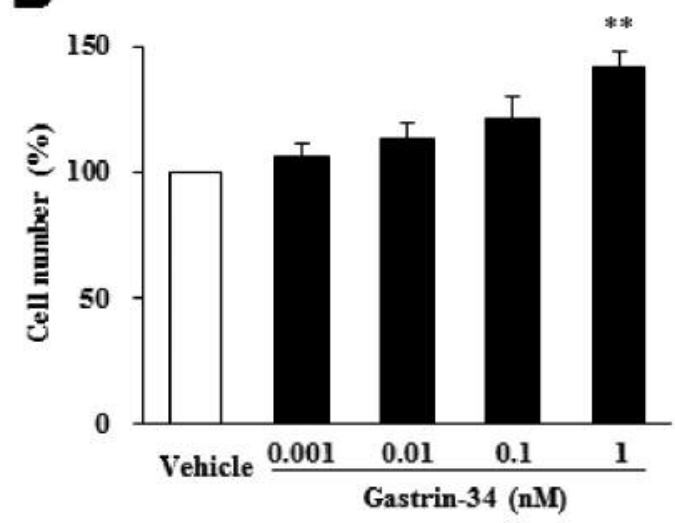

D

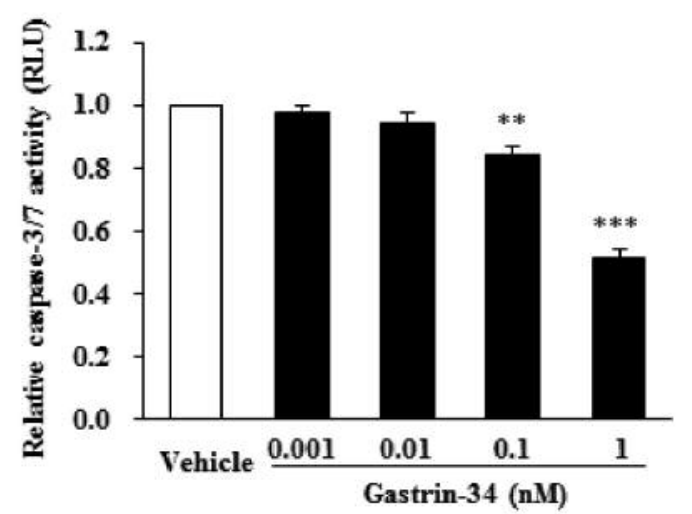

Figure 5. Effects of CCK2R ligands-induced anti-apoptosis in MIA PaCa-2/hCCK2R cells. (A) and (B) The cell number of MIA PaCa-2/hCCK2R cells was examined by MTS assay. Data were expressed as the mean \pm SEM of cell number (\%) in three independent experiments. The results compared with vehicle-treated groups were analyzed by Dunnett's test. (C) and (D) Apoptosis in MIA PaCa-2/hCCK2R cells was examined by the Caspase-Glo 3/7 assay. Data were expressed as the mean $\pm S E M$ of relative caspase-3/7 activity $(R L U)$ in three independent experiments. The results compared with vehicle-treated groups were analyzed by Dunnett's test. ${ }^{*} p<0.05,{ }^{*} p<0.01$ and $* * * p<0.001$. 

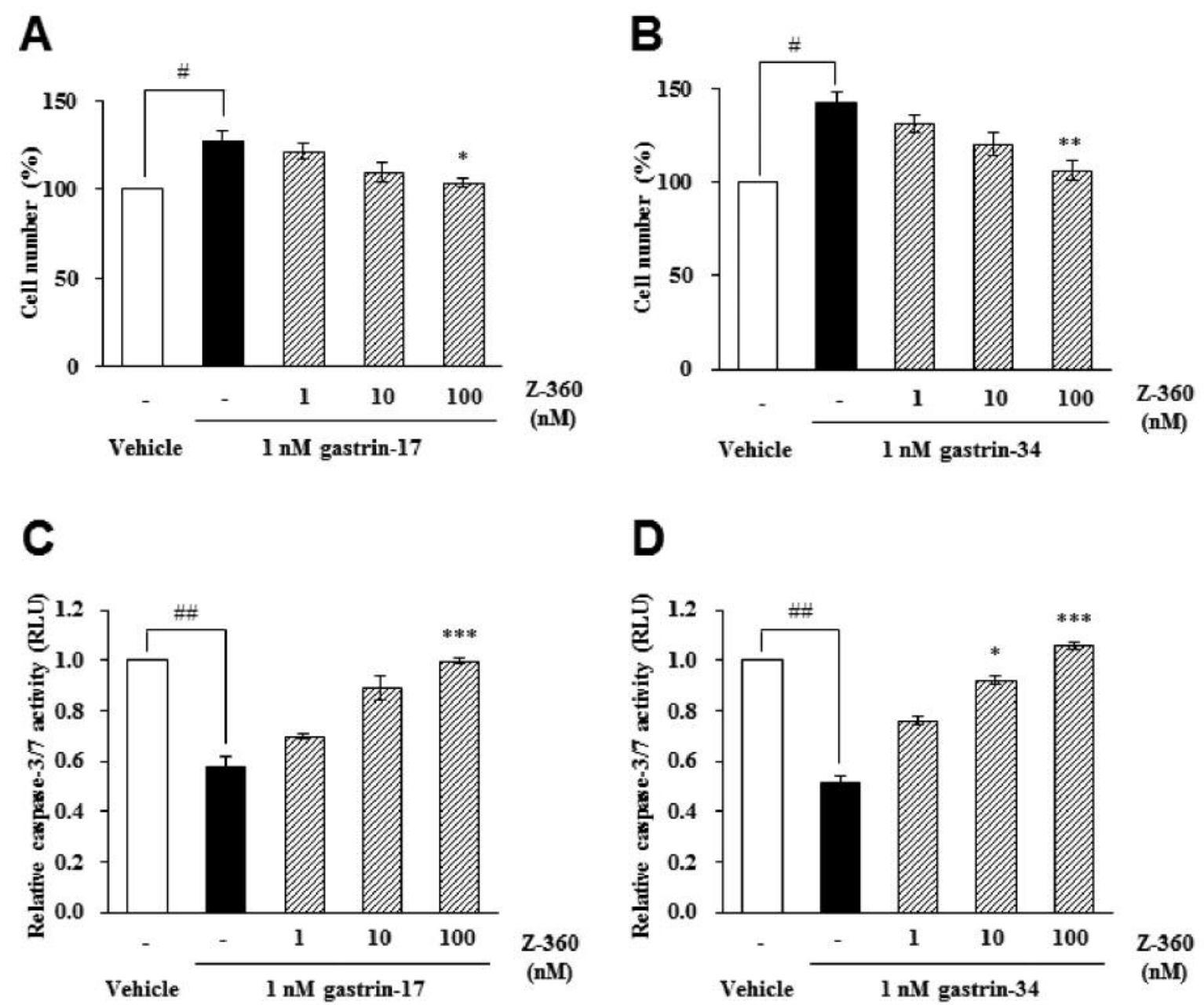

Z-360
$(\mathrm{nM})$

Figure 6. Inhibitory effects of Z-360 on CCK2R ligand-induced anti-apoptosis in MIA PaCa-2/hCCK2R cells. (A) and (B) The cell number in MIA PaCa-2/hCCK2R cells was examined by MTS assay. Data were expressed as the mean \pm SEM of cell number (\%) in three independent experiments. The results compared with vehicle-treated groups were analyzed by Student's t-test. The results compared with 1 nM gastrin-17- or gastrin-34treated groups were analyzed by Dunnett's test. $(C)$ and (D) Caspase-3/7 activity in MIA PaCa-2/hCCK2R cells was examined by the Caspase-Glo 3/7 assay. Data were expressed as the mean \pm SEM of caspase-3/7 activity (RLU) in three independent experiments. The results compared with vehicle-treated groups were analyzed by Student's t-test. ${ }^{\#} p<0.05$ and ${ }^{\# \#} p<0.01$. The results compared with 1 nM gastrin-17- or gastrin-34-treated groups were analyzed by Dunnett's test. ${ }^{*} p<0.05, * * p<0.01$ and $* * * p<0.001$.

type II cell-related genes (survivin, XIAP and Mcl-1), but not of type I cell-related genes (BIRC2 and BIRC3) in MIA $\mathrm{PaCa}-2$ pancreatic cells (Figure 3). This result matched that of a previous study demonstrating that pancreatic cancer cell lines are type II cells $(7,41-43)$. Thus, Z-360 may show an anti-tumor effect on cancers that mainly consist of type II cells, in addition to MIA PaCa-2 pancreatic cancer cells. As illustrated in Figure 3D, the combination of Z-360 and gemcitabine significantly down-regulated the gene expression of BIRC2 even though treatment with either agent did not decrease gene expression of BIRC2. Although there was no statistically significant difference between the vehicle group and gemcitabine-treated group in BIRC2 gene expression, gemcitabine tended to reduce the gene expression of BIRC2. Furthermore, a previous study reported that gemcitabine suppressed BIRC2 protein expression in the MIA PaCa-2 orthotopic xenograft mouse model (44). We, therefore, consider that gemcitabine substantially contributed to the results of combination treatment and that Z-360 hardly decreased the gene expression of BIRC2.

Combined therapies for pancreatic cancer have recently been developed, including 5-fluorouracil/leucovorin with irinotecan and oxaliplatin (FOLFIRINOX) and nabpaclitaxel. Although FOLFIRINOX and gemcitabine/nabpaclitaxel dramatically improve outcomes for patients with advanced pancreatic cancer, their use still poses challenges 
including recurrence and serious side effects (45). Thus, more effective and low-toxicity combination therapy is needed for patients with pancreatic cancer. While the mode of action of Z-360 is inhibition of CCK2R, gemcitabine as a nucleotide analogue is converted to difluorodeoxycytidine triphosphate (dFdCTP) by intra-cellular enzymes and incorporated into DNA, where it causes strand termination and in turn leads to apoptosis (46). Despite these differences in mechanism of action between Z-360 and gemcitabine, our study suggested that gemcitabine as well as Z-360 induced apoptosis and inhibited anti-apoptosis-related factors such as survivin, XIAP and Mcl-1 (Figures 2 and 3A-C). A previous report showed that gemcitabine down-regulated survivin and XIAP in the MIA PaCa-2 orthotopic xenograft model (47). We therefore consider that the combination of Z-360 and gemcitabine may have an additive effect concerning apoptosis. In fact, the combination of Z-360 and gemcitabine more potently inhibited the tumor growth than either agent alone (Figure 1). Furthermore, we previously reported that Z-360 rarely caused severe side effects in a phase Ib/IIa trial (34) while this agent showed a potent inhibitory effect against CCK2R (Figure 4B) (30). These results mean that combined therapy with Z-360 and gemcitabine could be more effective than monotherapy using either agent without causing serious side effects in pancreatic cancer patients.

Clinical trials have already been performed to evaluate the efficacy of the specific gastrin antibodies for advanced pancreatic carcinoma. Insegia, a vaccine that targets gastrin17 , significantly prolonged survival compared with placebo (48). These studies suggested that the inhibition of gastrindependent pathophysiological changes could be effective for the treatment of pancreatic carcinoma. The antibody that is raised against Insegia targets only gastrin-17 and does not cross-react with any of the other gastrin species, including gastrin-34 and CCK-8 (49). As previously reported, gastrin34 is a major molecular form of gastrins in plasma from hypergastrinaemic patients with gastric or pancreatic adenocarcinoma (50). In our study, we demonstrated that not only gastrin- 17 but also gastrin-34 shows an anti-apoptosis effect (Figures 4A and 5) and Z-360 dose-dependently inhibits these effects (Figures 4B and 6). Therefore, we expect that Z360 may inhibit the anti-apoptosis effects of both gastrin-17 and gastrin-34, while Insegia cannot inhibit the effect of gastrin-34, suggesting that the combination of Z-360 and Insegia has stronger anti-tumor effects than Insegia alone.

In conclusion, we demonstrated that Z-360 has an antitumor effect through the reduction of anti-apoptosis factors in a mouse model. Our results revealed that Z-360 canceled the anti-apoptosis effect of gastrin-34 as well as gastrin-17, suggesting that Z-360 might be clinically useful in the treatment of pancreatic cancer, particularly when combined with other anticancer drugs.

\section{Conflicts of Interest}

There are no conflicts of interest.

\section{Acknowledgments}

The Authors would like to thank Yugo Matsunaga, Takanori Sato, Masanao Kawachi, Katsunori Ito and Kenji Nagahama for technical support.

\section{References}

1 Bosetti C, Bertuccio P, Negri E, La vecchia C, Zeegers MP and Boffetta P: Pancreatic cancer: overview of descriptive epidemiology. Mol Carcinog 51(1): 3-13, 2012.

2 Siegel RL, Miller KD and Jemal A: Cancer statistics, 2016. CA Cancer J Clin 66(1): 7-30, 2016.

3 Rahib L, Smith BD, Aizenberg R, Rosenzweig AB, Fleshman JM and Matrisian LM: Projecting cancer incidence and deaths to 2030: the unexpected burden of thyroid, liver, and pancreas cancers in the United States. Cancer Res 74(11): 2913-2921, 2014.

4 Moore MJ, Goldstein D, Hamm J, Figer A, Hecht JR, Gallinger S, Au HJ, Murawa P, Walde D, Wolff RA, Campos D, Lim R, Ding K, Clark G, Nomikos TV, Ptasynski M and Parulekar W: Erlotinib plus gemcitabine compared with gemcitabine alone in patients with advanced pancreatic cancer: a phase III trial of the National Cancer Institute of Canada Clinical Trials Group. J Clin Oncol 25(15): 1960-1966, 2007.

5 Von Hoff DD, Ervin T, Arena FP, Chiorean EG, Infante J, Moore M, Seay T, Tjulandin SA, Wee Ma W, Saleh MN, Harris M, Reni M, Dowden S, Laheru D, Bahary N, Ramanathan RK, Tabernero J, Hidalgo M, Goldstein D, Van Cutsem E, Wei X, Iglesias J and Renschler MF: Increased survival in pancreatic cancer with nabpaclitaxel plus gemcitabine. N Engl J Med 369(18): 1691-1703, 2013.

6 Long J, Zhang Y, Yu X, Yang J, LeBrun DG, Chen C, Yao Q and Li M: Overcoming drug resistance in pancreatic cancer. Expert Opin Ther Targets 15(7): 817-828, 2011.

7 Westphal S and Kalthoff $\mathrm{H}$ : Apoptosis: targets in pancreatic cancer. Mol Cancer 2(1): 6, 2003.

8 Miyamoto Y, Hosotani R, Wada M, Lee J.-U., Koshiba T, Fujimoto K, Tsuji S, Nakajima S, Doi R, Kato M, Shimada Y and Imamura M: Immunohistochemical analysis of Bcl-2, Bax, Bcl-X, and Mcl-1 expression in pancreatic cancers. Oncology 56(1): 73-82, 1999.

9 Dong H, Qian D, Wang Y, Meng L, Chen D, Ji X and Fenget W: Survivin expression and serum levels in pancreatic cancer. World J Surg Oncol 13: 189, 2015.

10 Yang L, Cao Z, Yan H and Wood WC: Coexistence of high levels of apoptotic signaling and inhibitor of apoptosis proteins in human tumor cells: implication for cancer specific therapy. Cancer Res 63(20): 6815-6824, 2003.

$11 \mathrm{Li} \mathrm{S}$, Sun J, Yang J, Zhang L, Wang L, Wang X and Guo Z: XIAP expression is associated with pancreatic carcinoma outcome. Mol Clin Oncol 1(2): 305-308, 2013.

12 Shrikhande SV, Kleeff J, Kayed H, Keleg S, Reiser C, Giese T, Büchler MW, Esposito I and Friess H: Silencing of X-linked inhibitor of apoptosis (XIAP) decreases gemcitabine resistance of pancreatic cancer cells. Anticancer Res 26(5A): 3265-3273, 2006. 
13 Smith JP, Liu G, Soundararajan V, Mclaughlin PJ and Zagon IS: Identification and characterization of CCK-B/gastrin receptors in human pancreatic cancer cell lines. Am J Physiol 266(1 Pt 2): R277-283, 1994.

14 Caplin M, Savage K, Khan K, Brett B, Rode J, Varro A and Dhillon A: Expression and processing of gastrin in pancreatic adenocarcinoma. Br J Surg 87(8): 1035-1040, 2000.

15 Harris JC, Gilliam AD, Mckenzie AJ, Evans SA, Grabowska AM, Clarke PA, McWilliams DF and Watson SA: The biological and therapeutic importance of gastrin gene expression in pancreatic adenocarcinomas. Cancer Res 64(16): 5624-5631, 2004.

16 Smith JP, Fonkoua LK and Moody TW: The Role of Gastrin and CCK Receptors in Pancreatic Cancer and other Malignancies. Int J Biol Sci 12(3): 283-291, 2016.

17 Aly A, Shulkes A and Baldwin GS: Gastrins, cholecystokinins and gastrointestinal cancer. Biochim Biophys Acta 1704(1): 110, 2004.

18 Fourmy D, Gigoux V and Reubi JC: Gastrin in gastrointestinal diseases. Gastroenterology 141(3): 814-818.e1-3, 2011.

19 Szabó I, Rumi G, Bódis B, Németh P and Mózsik G: Gastrin and pentagastrin enhance the tumour proliferation of human stable cultured gastric adenocarcinoma cells. J Physiol Paris 94(1): 71$74,2000$.

20 Smith JP, Fantaskey AP, Liu G and Zagon IS: Identification of gastrin as a growth peptide in human pancreatic cancer. Am J Physiol 268(1 Pt 2): R135-141, 1995.

21 Dockray GJ: Topical review. Gastrin and gastric epithelial physiology. J Physiol (Lond) 518(Pt 2): 315-324, 1999.

22 Kanno N, Glaser S, Chowdhury U, Phinizy JL, Baiocchi L, Francis H, LeSage $\mathrm{G}$ and Alpini G: Gastrin inhibits cholangiocarcinoma growth through increased apoptosis by activation of $\mathrm{Ca}^{2+}$-dependent protein kinase $\mathrm{C}-\alpha$. J Hepatol 34(2): 284-291, 2001.

23 Selvik LK, Fjeldbo CS, Flatberg A, Steigedal TS, Misund K, Anderssen E, Doseth B, Langaas M, Tripathi S, Beisvag V, Lægreid A, Thommesen $\mathrm{L}$ and Bruland $\mathrm{T}$ : The duration of gastrin treatment affects global gene expression and molecular responses involved in ER stress and anti-apoptosis. BMC Genomics 14: 429, 2013.

24 Todisco A, Ramamoorthy S, Witham T, Pausawasdi N, Srinivasan S, Dickinson CJ, Askari FK and Krametter D: Molecular mechanisms for the antiapoptotic action of gastrin. Am J Physiol Gastrointest Liver Physiol 280(2): G298-307, 2001.

25 Subbannayya Y, Anuja K, Advani J, Ojha UK, Nanjappa V, George B, Sonawane A, Kumar RV, Ramaswamy G, Pandey A, Somani BL and Raju R: A network map of the gastrin signaling pathway. J Cell Commun Signal 8(2): 165-170, 2014.

26 Wong $\mathrm{HH}$ and Lemoine NR: Pancreatic cancer: molecular pathogenesis and new therapeutic targets. Nat Rev Gastroenterol Hepatol 6(7): 412-422, 2009.

27 Mandair KK, Towner P, Stamford IF, Morris JD, Harper E, Benjamin IS and Tavares IA: Cholecystokinin receptors in human pancreatic cancer cell lines. Eur J Cancer 34(9): 1455-1459, 1998.

28 Smith JP, Shih A, Wu Y, Mclaughlin PJ and Zagon IS: Gastrin regulates growth of human pancreatic cancer in a tonic and autocrine fashion. Am J Physiol 270(5 Pt 2): R1078-1084, 1996.

29 Chau I, Cunningham D, Russell C, Norman AR, Kurzawinski T, Harper P, Harrison P, Middleton G, Daniels F, Hickish T, Prendeville J, Ross PJ, Theis B, Hull R, Walker M, Shankley N, Kalindjian B, Murray G, Gillbanks A and Black J: Gastrazole
(JB95008), a novel CCK2/gastrin receptor antagonist, in the treatment of advanced pancreatic cancer: results from two randomised controlled trials. Br J Cancer 94(8): 1107-1115, 2006.

30 Kawasaki D, Emori Y, Eta R, Iino Y, Hamano H, Yoshinaga K, Tanaka T, Takei M and Watson SA: Effect of Z-360, a novel orally active CCK-2/gastrin receptor antagonist on tumor growth in human pancreatic adenocarcinoma cell lines in vivo and mode of action determinations in vitro. Cancer Chemother Pharmacol 61(5): 883-892, 2008.

31 Grabowska AM, Morris TM, Mckenzie AJ, Kumari R, Hamano $\mathrm{H}$, Emori Y, Yoshinaga K and Watson SA: Pre-clinical evaluation of a new orally-active CCK-2R antagonist, Z-360, in gastrointestinal cancer models. Regul Pept 146(1-3): 46-57, 2008.

32 Boyce M, Lloyd KA and Pritchard DM: Potential clinical indications for a CCK2 receptor antagonist. Curr Opin Pharmacol 31: 68-75, 2016.

33 Miura N, Yoneta T, Ukawa H, Fujkuda Y, Eta R, Mera Y, Omata T, Kinomoto T, Kurimoto T and Itoh Z: Pharmacological profiles of Z-360, a novel CCK-B/Gastrin (CCK2) receptor antagonist with excellent oral potency. Gastroenterology 120(5): A311, 2001.

34 Meyer T, Caplin ME, Palmer DH, Valle JW, Larvin M, Waters JS, Coxon F, Borbath I, Peeters M, Nagano E and Kato H: A phase Ib/IIa trial to evaluate the CCK2 receptor antagonist Z360 in combination with gemcitabine in patients with advanced pancreatic cancer. Eur J Cancer 46(3): 526-533, 2010.

35 Rengifo-cam W and Singh P: Role of progastrins and gastrins and their receptors in GI and pancreatic cancers: targets for treatment. Curr Pharm Des 10(19): 2345-2358, 2004.

36 Singh P: Role of Annexin-II in GI cancers: interaction with gastrins/progastrins. Cancer Lett 252(1): 19-35, 2007.

37 Pritchard DM, Berry D, Przemeck SM, Campbell F, Edwards SW and Varro A: Gastrin increases mcl-1 expression in type I gastric carcinoid tumors and a gastric epithelial cell line that expresses the CCK-2 receptor. Am J Physiol Gastrointest Liver Physiol 295(4): G798-805, 2008.

38 Konturek PC, Kania J, Kukharsky V, Ocker S, Hahn EG and Konturek SJ: Influence of gastrin on the expression of cyclooxygenase-2, hepatocyte growth factor and apoptosisrelated proteins in gastric epithelial cells. J Physiol Pharmacol 54(1): 17-32, 2003.

39 Fino KK, Matters GL, Mcgovern CO, Gilius EL and Smith JP: Downregulation of the CCK-B receptor in pancreatic cancer cells blocks proliferation and promotes apoptosis. Am J Physiol Gastrointest Liver Physiol 302(11): G1244-1252, 2012.

40 Barnhart BC, Alappat EC and Peter ME: The CD95 type I/type II model. Semin Immunol 15(3): 185-193, 2003.

41 Scaffidi C, Fulda S, Srinivasan A, Friesen C, Li F, Tomaselli KJ, Debatin KM, Krammer PH and Peter ME: Two CD95 (APO1/Fas) signaling pathways. EMBO J 17(6): 1675-1687, 1998.

42 Hinz S, Trauzold A, Boenicke L, Sandberg C, Beckmann S, Bayer E, Walczak H, Kaltho $\mathrm{H}$ and Ungefroren $\mathrm{H}$ : Bcl-XL protects pancreatic adenocarcinoma cells against CD95- and TRAIL-receptor-mediated apoptosis. Oncogene 19(48): 54775486, 2000

43 Trauzold A, Wermann H, Arlt A, Schütze S, Schäfer H, Oestern S, Röder C, Ungefroren H, Lampe E, Heinrich M, Walczak H and Kaltho H: CD95 and TRAIL receptor-mediated activation of protein kinase $\mathrm{C}$ and NF-kappaB contributes to apoptosis resistance in ductal pancreatic adenocarcinoma cells. Oncogene 20(31): 4258-4269, 2001 
44 Kunnumakkara AB, Guha S, Krishnan S, Diagaradjane P, Gelovani J and Aggarwal BB: Curcumin potentiates antitumor activity of gemcitabine in an orthotopic model of pancreatic cancer through suppression of proliferation, angiogenesis, and inhibition of nuclear factor-kB-regulated gene products. Cancer Res 67(8): 3853-3861, 2007.

45 Chand S, O'hayer K, Blanco FF, Winter JM and Brody JR: The Landscape of Pancreatic Cancer Therapeutic Resistance Mechanisms. Int J Biol Sci 12(3): 273-282, 2016

46 Voutsadakis IA: Molecular predictors of gemcitabine response in pancreatic cancer. World J Gastrointest Oncol 3(11): 153-164, 2011.

47 Harikumar KB, Kunnumakkara AB, Sethi G, Diagaradjane P, Anand P, Pandey MK, Gelovani J, Krishnan S, Guha S and Aggarwal BB: Resveratrol, a multitargeted agent, can enhance antitumor activity of gemcitabine in vitro and in orthotopic mouse model of human pancreatic cancer. Int J Cancer 127(2): 257-268, 2010.
48 Gilliam AD, Watson SA, Henwood M, McKenzie AJ, Humphreys JE, Elder J, Iftikhar SY, Welch N, Fielding J, Broome P and Michaelil D: A phase II study of G17DT in gastric carcinoma. Eur J Surg Oncol 30(5): 536-543, 2004.

49 Smith AM, Justin T, Michaeli D and Watson SA: Phase I/II study of G17-DT, an anti-gastrin immunogen, in advanced colorectal cancer. Clin Cancer Res 6(12): 4719-4724, 2000.

50 Rehfeld JF and Goetze JP: Gastrin vaccination against gastrointestinal and pancreatic cancer. Scand J Gastroenterol 41(1): 122-123, 2006.
Received June 6, 2017

Revised June 29, 2017

Accepted June 30, 2017 